\title{
Adenoma corticoadrenal, presentándose como un incidentaloma. Presentación de un caso y revisión de la literatura
}

\author{
Sandra Herrera L. ${ }^{1}$, Carolaine Ortega A. ${ }^{1}$, Miguel Aguilar S. ${ }^{1}$ y Hugo Corrales S. ${ }^{1}$
}

\section{Adrenocortical adenoma, presenting as an incidentaloma.}

\section{Case report and literature review}

Objective: We present a clinical case with diagnosis of an asymptomatic nonfunctional adrenal incidentaloma, in which we discuss the clinical implications and the approach. Clinical case: Male patient, 53 years old with an accidental sonographic finding, characterized by a hypoechoic image of well-defined contours in the right adrenal gland of less than $2 \mathrm{~cm}$. The hormonal test showed no adrenal hyperfunctioning. Laparoscopic adrenalectomy technique is performed with 4 trocars with complete excision of the lesion. The patient presented good postoperative evolution. Results: The pathology study showed a well-defined and benign tumor lesion of the adrenal gland, being similar to the fascicular zone and cortical hyperplasia next to it. The diagnosis is a non-functioning adenoma of the adrenal gland derived from the fascicular zone. Conclusion: Given the finding of an adrenal mass greater than $1 \mathrm{~cm}$ mass corresponds perform a hormonal identification and risk assessment of malignancy in patients, which with imaging parameters (echogenicity, bilateralism and the adjacent commitment) and symptoms presented allow to identify the complications in the management and prognosis of the patient. The differential diagnosis of adrenal adenomas is based on the hormonal evaluation, radiological knowledge and the commitment of the injury.

Key words: adrenal tumors; adrenal incidentaloma; laparoscopic adrenalectomy.

\section{Resumen}

Objetivo: Presentamos un caso clínico con diagnóstico de incidentaloma adrenal no funcionante asintomático y analizamos las implicaciones clínicas y el abordaje realizado. Caso clínico: Se reporta el caso de un masculino de 53 años, asintomático, con hallazgo ecográfico accidental de imagen hipoecoica de contornos bien definidos en la glándula suprarrenal derecha que presentó incremento en su tamãno. Su estudio hormonal fue negativo para hiperfunción adrenal. Resultados: Se realizó suprarrenalectomía laparoscópica con técnica de 4 trocares con resección completa de la lesión. El paciente presentó buena evolución posquirúrgica. El estudio anatomopatológico concluyó el diagnóstico de adenoma corticoadrenal no funcionante. Conclusión: Ante el hallazgo de una masa adrenal mayor de $1 \mathrm{~cm}$ corresponde realizar una identificación hormonal y una evaluación del riesgo de malignidad en los pacientes, los cuales, junto con parámetros imagenológicos y los síntomas presentados, permitirán definir las complicaciones en el manejo y el pronóstico del paciente. El diagnóstico diferencial de los adenomas adrenales está basado en la identificación hormonal, el conocimiento radiológico y el grado de compromiso de la lesión. El abordaje laparoscópico es de elección en las lesiones pequeñas y sin sospecha de malignidad.

Palabras clave: tumores suprarrenales; incidentaloma suprarrenal; suprarrenalectomía laparoscópica.

\section{Introducción}

Se define como incidentaloma adrenal una masa suprarrenal descubierta de manera casual, durante una evaluación por imágenes realizada por una indicación no vinculada con la evaluación de dicha glándula ${ }^{1}$. Dentro este grupo, se excluyen aquellos pacientes asintomáticos con predisposición genética para tumores suprarrenales que motiven el estudio imagenológico, con cáncer extraadrenal en proceso de estadificación o con manifestaciones clínicas sugestivas de disfunción suprarrenal ${ }^{1,2}$. Son hallados
'Grupo de Investigación Centro de Investigaciones Biomédicas, Facultad de Medicina, Universidad de Cartagena, Cartagena, Colombia.

Recibido el 31 de enero de 2017; aceptado el 12 de febrero de 2017.

Correspondencia a:

Sandra Herrera L.

sherreral1@hotmail.com 
en el 4-5\% de las TC (tomografías computarizadas) y se presentan hasta en un $9 \%$ de las autopsias ${ }^{1,3}$. $\mathrm{Su}$ incidencia depende de la edad de los pacientes, encontrándose aproximadamente el $0,2 \%$ en la tercera década de la vida e incrementándose hasta el $7 \%$ a los 70 años, sin distinción entre hombres y mujeres $^{1,2}$.

De todos los incidentalomas adrenales, el $70 \%$ corresponden a adenomas no funcionantes, el 5-16\% a adenomas funcionantes, el $6 \%$ a feocromocitomas, el $5 \%$ a carcinomas adrenocorticales, el $2 \%$ a metástasis, y el resto, a otros tipos de lesiones (mielolipomas, hematomas, quistes o linfoma $)^{1,2,4,5}$.

\section{Caso clínico}

Masculino de 53 años de edad, asintomático, que fue remitido por el hallazgo ecográfico accidental de una masa hipoecoica, bien delimitada en la glándula suprarrenal derecha, de $19 \mathrm{~mm}$ de diámetro. El paciente refirió antecedentes de episodios aislados de hipertensión arterial, considerados como hipertensión de bata blanca, definida como registros de presión elevada en la consulta médica sin evidencia de cifras tensionales iguales o mayores fuera de este medio. También refirió hiperplasia prostática benigna, rinitis alérgica y varicocele bilateral corregido quirúrgicamente. Negó diabetes, episodios de diaforesis, taquicardia, cefalea, cambios de peso, alteraciones del vello corporal o del porcentaje de masa muscular.

Al examen físico se encontró normotenso, sin fenotipo específico, abdomen blando, depresible, no doloroso, sin masas ni visceromegalias. La radiografía de abdomen simple y la tomografía computarizada confirmaron lesión suprarrenal derecha de 32 x $25 \mathrm{~mm}$, de contornos definidos y densidad heterogénea, con porciones internas de contenido graso (Figura 1).

El estudio endocrinológico fue negativo para hiperfunción hormonal, con un valor de cortisol de $12 \mathrm{~g} / \mathrm{dl}$. De igual forma se obtuvieron resultados normales en el perfil lipídico y electrólitos séricos, exceptuando los niveles de glucosa, que resultaron levemente incrementados: $110 \mathrm{mg} / \mathrm{dl}$.

En vista del incremento del tamaño de la lesión se decidió realizar suprarrenalectomía vía laparoscópica derecha, con técnica de 4 trocares subcostales con resección de la lesión completa. El estudio anatomopatológico describió glándula suprarrenal derecha, con masa de $4 \times 4 \times 3 \mathrm{~cm}$, peso de $29 \mathrm{~g}$, bien delimitada, amarillenta, blanda, encapsulada (Figura 2). Al examen microscópico se observó un tumor benigno, representado por una proliferación de células grandes de citoplasma amplio, que simulan la zona fasciculada, sin atipias, necrosis, ni incremento en el número de mitosis, positivas para Melan-A, con un índice de proliferación medido con Ki67 del 0-2\%, confirmando la benignidad del tumor. No hubo reactividad para cóctel de queratinas, cromogranina, sinaptofisina, ni S100, descartándose el origen neuroendocrino. Se concluyó el diagnóstico de adenoma suprarrenal, no funcionante, originario de zona fasciculada, completamente resecado (Figura 3).

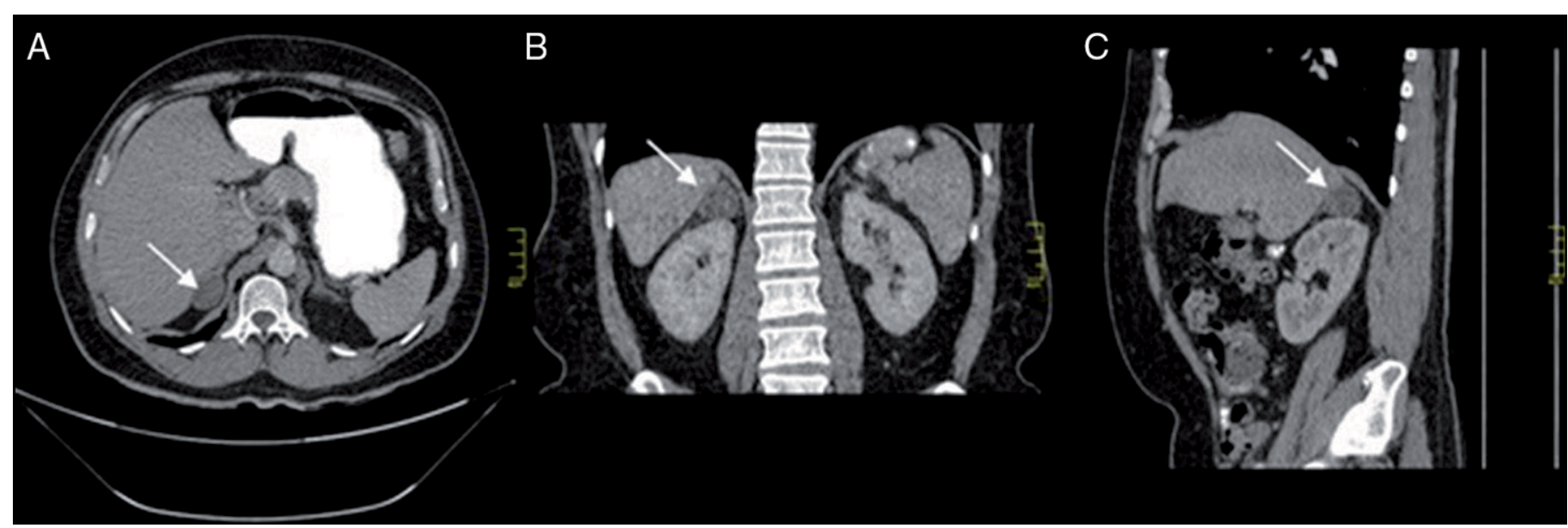

Figura 1. Tomografía computarizada de abdomen donde se observa tumoración sólida en la glándula suprarrenal derecha de $32 \times 25 \mathrm{~mm}$, de contornos definidos y densidad heterogénea, señalada con flechas blancas. A) Corte axial. B) Reconstrucción coronal. C) Reconstrucción lateral. 


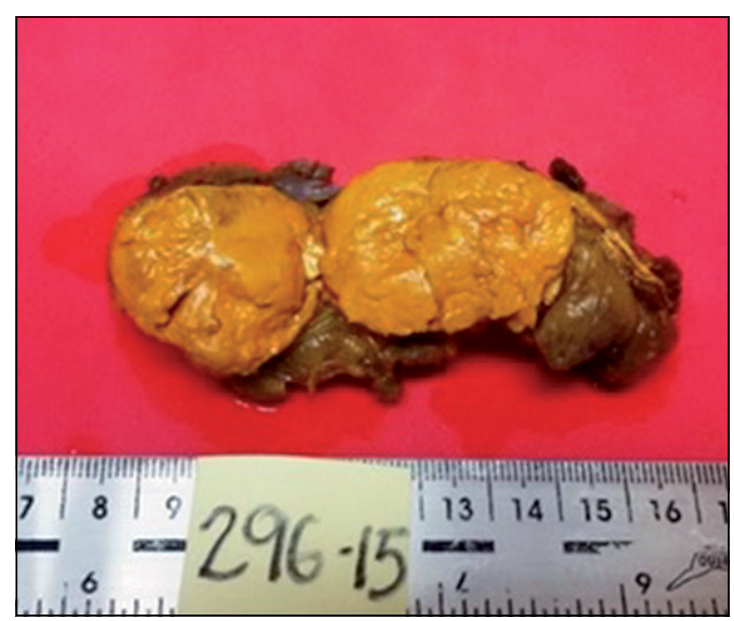

Figura 2. Pieza de resección de la suprarrenalectomía derecha que muestra lesión nodular de aproximadamente $4 \mathrm{~cm}$ adyacente a tejido adrenal sano.

\section{Discusión}

Dentro del espectro de presentación, el 70-80\% de los incidentalomas adrenales son adenomas corticales no funcionantes benignos ${ }^{5}$. Los hallazgos muestran un pico de incidencia entre la quinta y la séptima década de la vida, con una media de 55 años, y una prevalencia mayor en mujeres, aunque en los hombres existe mayor probabilidad de malignidad $^{3}$. Las masas adrenales son detectadas en la glándula suprarrenal derecha en el $50-60 \%$ de los casos y en la izquierda en el 30-40\%. El hallazgo de masas suprarrenales bilaterales se da en el 10-15\% de los casos, lo cual es generalmente sugestivo de metástasis, infiltraciones extraadrenales o hiperplasias focales o congénitas de la glándula ${ }^{6}$.

Ante el hallazgo de una masa adrenal mayor de $1 \mathrm{~cm}$ corresponde realizar una identificación hormo- nal y posterior evaluación del riesgo de malignidad de estos pacientes ${ }^{5}$; lo anterior es importante, no solo porque las tumoraciones suprarrenales funcionales constituyen una indicación quirúrgica, sino porque pueden requerir una preparación farmacológica preoperatoria especial para disminuir la morbimortalidad relacionada con el evento quirúrgico, como en el caso del feocromocitoma ${ }^{7}$.

El examen físico cobra importancia para determinar signos y síntomas clínicos sugestivos de síndrome de Cushing, hiperaldosteronismo, feocromocitoma, o cambios resultantes de exceso de hormonas sexuales (virilización y feminización) ${ }^{8}$. Aunque existe el debate, actualmente la Red Europea para estudios de tumores adrenales recomienda realizar estudios hormonales incluso en pacientes asintomáticos y sin riesgo de malignidad, ya que se ha demostrado la asociación de adenomas no funcionantes con síndromes subclínicos y alteraciones metabólicas a la hora del diagnóstico ${ }^{4,8}$. Sin embargo, estos estudios no se realizan en pacientes con imágenes sugestivas de adenomas no funcionantes como mielolipomas o quistes 9 . Es importante diferenciar las características clínicas e imagenológicas de los adenomas funcionantes, ya que son el diagnóstico principal a descartar en el abordaje de un incidentaloma. En la Tabla 1 se resumen aspectos clave diagnósticos de estas neoplasias ${ }^{1,2,5,7}$.

En algunos casos es válido realizar pruebas de cribado cuando existe la sospecha de síndrome subclínico. El síndrome de Cushing es el más frecuente de ellos, con una prevalencia del $12-24 \%^{3}$; su definición incluye un hipercortisolismo bioquímico, sin datos clínicos, pero con al menos 2 pruebas anormales que indican sobreproducción endógena de corti$\mathrm{sol}^{7}$. Aunque los pacientes con masas productoras y manifestaciones clínicas tienen un diagnóstico y manejo más específico por su compromiso metabólico,

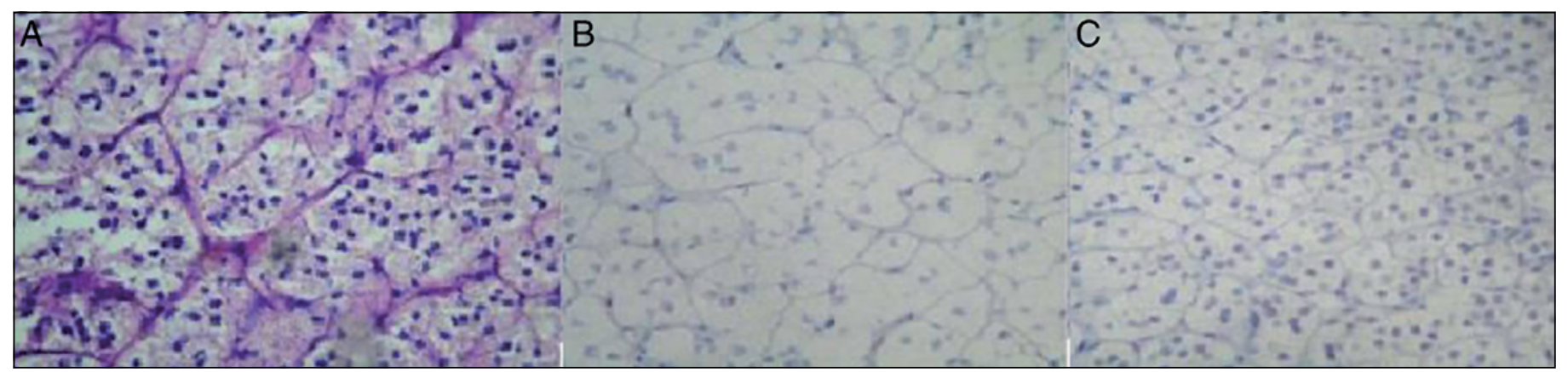

Figura 3. Imagen histológica de la pieza de la suprarrenalectomía: A) tinción de hematoxilina y eosina que muestra células sin atipia dispuestas en un patrón organoide (40x); B y C) inmunohistoquímicas para cromogranina y sinaptofisina que muestran negatividad (10×). 


\section{CASO CLÍNICO}

Tabla 1. Características clínicas de adenomas corticales funcionantes

\begin{tabular}{|c|c|c|c|}
\hline Características & Síndrome de Cushing & Feocromocitoma & Aldosteronoma \\
\hline Frecuencia & $30-40 \%$ & $10-20 \%$ & $4-5 \%$ \\
\hline HTA sistémica & Elevada leve/moderada & Elevada moderada/severa & Elevada leve/moderada \\
\hline Hiperglucemia & $42 \%$ & $33 \%$ & $32 \%$ \\
\hline Claves diagnósticas clínicas & Síndrome metabólico asociado & $\begin{array}{l}\text { Cuadro simpático: cefalea, } \\
\text { diaforesis y palpitaciones }\end{array}$ & $\begin{array}{l}\text { Hipocalemia, debilidad muscular } \\
\text { y poliuria }\end{array}$ \\
\hline
\end{tabular}

no está claro si los incidentalomas no productores y subclínicos tienen potencial para comprometer la salud del paciente a largo plazo, por lo que sería clave realizar una estratificación del riesgo para optimizar una intervención individualizada ${ }^{9-11}$.

El tamaño de la lesión es muy importante a la hora de elegir la conducta terapéutica; las masas mayores de $6 \mathrm{~cm}$ deben ser removidas, a pesar de ser no funcionantes, según el perfil hormonal, pues tienen un alto potencial maligno ${ }^{7}$. Generalmente, las lesiones que son menores de $4 \mathrm{~cm}$ y que son definidas como de bajo riesgo por criterios de imagen es improbable que tengan potencial maligno, y pueden manejarse de manera conservadora con seguimiento imagenológico cada 6 a 12 meses junto con evaluación del perfil hormonal; si el tamaño de la masa aumenta más de $1 \mathrm{~cm}$ o si se evidencia producción hormonal, se debe practicar resección ${ }^{9,12}$. En caso de que no puedan ser determinadas correctamente las características tumorales por el análisis imagenológico, se recomienda realizar el manejo quirúrgico y posterior estudio anatomopatológico ${ }^{5,12,13}$.

Para las lesiones no funcionantes, la evaluación del crecimiento en los estudios controles muestra que el $5-25 \%$ de las lesiones menores de $6 \mathrm{~cm}$ aumentarán de tamaño, y el riesgo de malignidad se estima de 1 en $1.000^{3,9}$.

La punción con aguja fina (PAAF) está indicada en la caracterización de lesiones que crecen, ante hallazgos radiológicos equívocos en función del contexto clínico y cuando hay alta sospecha de metástasis; la ecografía no es de utilidad en estos $\operatorname{casos}^{2,13}$. Generalmente, las lesiones que son menores de $4 \mathrm{~cm}$ y son definidas como de bajo riesgo por criterios de imagen e improbable que tengan potencial maligno no son resecadas ${ }^{12}$. Este tipo de manejo también se opta en adenomas funcionales, lógicamente teniendo en cuenta el riesgo de manifestaciones adversas durante la cirugía ${ }^{5,12,13}$.

La vía de abordaje de elección es la laparoscópica para los tumores benignos, funcionantes y no funcionantes, menores de $8-10 \mathrm{~cm}$, ya que aporta las ventajas de la cirugía mínimamente invasiva, como la pronta recuperación del paciente. Para las lesiones mayores de $10 \mathrm{~cm}$, y para aquellas en las que se sospeche malignidad, se recomienda la vía abierta, con el fin de lograr márgenes libres adecuados ${ }^{9,14,15}$.

En el presente caso se sugirió el diagnóstico de un adenoma benigno no funcionante, los cuales corresponden al 70-80\% de los incidentalomas adrenales 5 . Sin embargo, imagenológicamente se observó crecimiento comparativo de la lesión, por lo cual se decidió su extracción vía laparoscópica. Menos probable resultó la posibilidad de que se tratara de una lesión maligna como el carcinoma corticosuprarrenal porque este se presenta bilateralmente, es heterogéneo en TC y secretor de precursores de esteroides sexuales, que rara vez puede causar virilización o feminización $^{12}$; la sospecha de lesión metastásica se establece cuando estos hallazgos estén acompañados de pérdida de peso o síntomas constitucionales ${ }^{5,7-9}$.

El adenoma cortical típico es una lesión nodular bien delimitada de hasta $2,5 \mathrm{~cm}$ de diámetro que expande la suprarrenal; cuando son funcionantes, se relacionan con atrofia del parénquima circundante. Suelen tener apariencia amarilla-marrón en la superficie por la presencia de lípidos; microscópicamente están formados por células similares a las de la corteza suprarrenal normal, y sus núcleos tienden a ser más pequeños ${ }^{3,14,16}$.

Concluimos que el diagnóstico diferencial de un incidentaloma suprarrenal requiere de los conoci- 
mientos anatómico y funcional de esta glándula, que, relacionados con la clínica, la evaluación hormonal e imágenes, pueden predecir el riesgo de malignidad y/o complicaciones a presentar en el paciente, por lo que debemos estar alertas en cuanto al riesgo-beneficio del manejo conservador.

\section{Responsabilidades éticas}

Protección de personas y animales. Los autores declaran que para esta investigación no se han realizado experimentos en seres humanos ni en animales.

Confidencialidad de los datos. Los autores declaran que han seguido los protocolos de su centro de trabajo sobre la publicación de datos de pacientes.

Derecho a la privacidad y consentimiento informado. Los autores declaran que en este artículo no aparecen datos de pacientes.

\section{Financiación}

Los autores declaran no haber recibido ninguna financiación para la realización de este trabajo.

\section{Conflicto de intereses}

Los autores declaran no tener ningún conflicto de intereses.

\section{Bibliografía}

1. Chervin R, Herrera J, Juri A, Pardes E, Surraco ME. Mesa 1: Incidentaloma suprarrenal. Tercer Consenso Argentino sobre Patologías Endocrinológicas. Federación Argentina de Sociedades de Endocrinología; Buenos Aires. Rev Argent Endocrinol Metab. 2009;46:55-64.

2. Oliveira Caiafa R, Salvador Izquierdo R, Buñesch Villalba L, Sebastià Cerqueda MC, Nicolau Molina C. Manejo y diagnóstico del incidentaloma suprarrenal. Radiología. 2011;53:516-30.

3. Barzon L, Sonino N, Fallo F, Palu G, Boscaro M. Prevalence and natural history of adrenal incidentalomas. Eur J Endocrinol. 2003;149:273-85.

4. Linos D. Adrenal incidentaloma (adrenaloma). Hormones (Athens). 2003;2:12-21

5. Arlt W. Disorders of the Adrenal Cortex. En: Harrison's principles of internal medicine. 18th ed. New York: McgrawHill; 2011. p. 2940-61.

6. Anagnostis P, Karagiannis A, Tziomalos K, Kakafika AI, Athyros VG, Mikhailidis
DP. Adrenal incidentaloma: A diagnostic challenge. Hormones (Athens). 2009;8:163-84.

7. Rivera-Hernández A, Díaz-Zavala F. Incidentaloma adrenal. Presentación de un caso y revisión de la literatura. Rev Endocrinol Nutr. 2013;21:182-6.

8. Clinical presentation and evaluation of adrenocortical tumors. UpToDate; 2015 [consultado 09 Sep 2015]. Disponible en: http://www.uptodate.com/contents/ clinical-presentationand-evaluation-ofadrenocortical-tumors.

9. Grumbach MM, Biller BM, Braunstein GD, Campbell KK, Carney JA, Godley PA, et al. Management of the clinically inapparent adrenal mass (incidentaloma). Ann Intern Med. 2003;138:424-9.

10. Moreno A, Santiago P, Sánchez C, Martínez P. Protocolo de estudio y tratamiento del incidentaloma adrenal Una aproximación actualizada. Seminario Médico. 2003;55:93-8.

11. Androulakis II, Kaltsas G, Piaditis G, Grossman AB. The clinical significance of adrenal incidentalomas. Eur J Clin Invest. 2011;41:552-60
12. Marcondes JA, Curi DD, Matsuzaki CN Barcellos CR, Rocha MP, Hayashida $\mathrm{SA}$, et al. Ovarian hyperthecosis in the context of an adrenal incidentaloma in a postmenopausal woman. Arq Bras Endocrinol Metabol. 2008;52:1184-8. Epub 2008/12/17.

13. Allen BC, Francis IR. Adrenal imaging and intervention. Radiol Clin North Am. 2015;53:1021-35.

14. Gac EP, Cabané TP, Jans BJ, Marambio GA, Díaz BM, Araya QV, et al. Manejo quirúrgico del incidentaloma suprarrenal. Rev Chil Cir. 2012;64:25-31.

15. Lamas S, Pujol J, García-Barrasa A, Mora L, Moreno P, Rafecas A, et al. Adrenalectomía laparoscópica: causas de conversión, experiencia personal y revisión de la bibliografía. Cir Esp. 2004; 75:18-22.

16. López JE, Marcano Torres M, Sukerman E, López Salazar JE, López Salazar Y, Urbaneja H, et al. Tumores suprarrenales: presentación de 8 pacientes con estudios bioquímicos e histopatológicos. Gac Méd Caracas. 2003;111:30-45 